\title{
Fragilización por Hidrógeno de los Aceros API 5L X60 y API 5L X80
}

\author{
Bruno A. Araújo ${ }^{(1)}$, Jorge A. Palma ${ }^{(1)}$, Eudésio O. Vilar ${ }^{(2)}$ y Antonio A. Silva ${ }^{(3)}$ \\ Universidad Federal de Campina Grande, Av. Aprígio Veloso, 882 - Bodocongó, \\ C. Grande, PB-Brasil. (1) Doctorado en Ciencias e Ingeniería de Materiales \\ (e-mail: bengmec@yahoo.com.br, jcarrasco@cct.ufcg.edu.br); (2) Unidad Académica de \\ Ingeniería Química (e-mail: vilar@deq.ufcg.edu.br); (3) Unidad Académica de Ingeniería \\ Mecánica (e-mail: almeida@dem.ufcg.edu.br)
}

Recibido Feb. 02, 2011; Aceptado Mar. 29, 2011; Versión Final recibida May. 20, 2011

\begin{abstract}
Resumen
Se ha determinado el comportamiento mecánico de los aceros API $5 \mathrm{~L}$ grados X60 y X80, fragilizados por hidrógeno ambiental y por hidrógeno interno. El potencial y la densidad de corriente para la generación del hidrógeno fueron determinados por polarización potenciodinámica, y el tiempo de saturación estimado a partir de datos de difusividad y solubilidad obtenidos en ensayos de permeación con una célula electroquímica. La carga de hidrógeno para reproducir las condiciones de la fragilización por hidrógeno ambiental y fragilización por hidrógeno interno fue realizada generando un gradiente de concentración para el primer caso, y generando gradientes de concentración y de tensiones para el segundo. Luego de los ensayos de tracción se verificò que, en las condiciones definidas en este trabajo, el modo de fractura en ambos tipos de fragilización fue predominantemente dúctil. Ademàs, los dos aceros mostraron una susceptibilidad baja o moderada a la fragilización por hidrógeno ambiental, no siendo susceptibles a la fragilización por hidrógeno interno.
\end{abstract}

\section{Hydrogen Embrittlement of API 5L X60 and API 5L X80 Steels}

\begin{abstract}
The mechanical behavior of the API $5 \mathrm{~L} X 60$ and $X 80$ grade steels under environmental hydrogen embrittlement effect and internal hydrogen embrittlement effect was determined. The potential and current density for hydrogen generation were determined by potentiodynamic polarization. The saturation time was estimated from diffusivity and solubility data, obtained from permeation tests with an electrochemical cell. The hydrogen charge to reproduce the conditions of the environmental hydrogen embrittlement and the internal hydrogen embrittlement was performed by generating a concentration gradient in the first case, and a concentration and stress gradients in the second case. After the tensile tests it was verified that in the defined tests conditions, the fracture mode in both types of embrittlement was predominantly ductile. Also, both steels showed low or moderate susceptibility to environmental hydrogen embrittlement, not being susceptible to internal hydrogen embrittlement.
\end{abstract}

Keywords: hydrogen embrittlement, potentiodynamic polarization, API 5 L X60 steel, API 5L X80 steel 


\section{INTRODUCCIÓN}

En la industria del petróleo cada año son destinados millones de dólares a la reparación o reemplazo de tubos que presentan fallas provocadas por procesos de degradación de las propiedades mecánicas del acero asociados con el ambiente de trabajo de esas estructuras. Un fenómeno que se cita con frecuencia es la Fragilización por Hidrógeno - FH, clasificada en Fragilización por Hidrógeno Ambiental - FHA y Fragilización por Hidrógeno Interno - FHI (ASM, 1987). La FHA ocurre por la adsorción de hidrógeno molecular generado en una atmósfera hidrogenada, o por una reacción de corrosión, y su absorción en el reticulado cristalino luego de su disociación a la forma atómica. La FHI ocurre en ausencia de una atmósfera hidrogenada y es causada por el hidrógeno que ingresa en el reticulado durante la producción del acero y en algunos de los procesos de fabricación de las estructuras, como la soldadura, antes de las pruebas o del inicio del servicio. Ese ingreso es facilitado debido a que la solubilidad del hidrógeno en el metal fundido es mucho más alta que cuando está en estado sólido. Una vez en el reticulado, y en presencia de tensiones, el hidrógeno fragiliza el material luego de un período de tiempo que es función de la concentración, temperatura y del estado tensional de la matriz (Woodtli y Kieselbach, 2000; Eliaz et al., 2002). Los metales y aleaciones metálicas registran comportamientos diferentes bajo esas dos condiciones. Kuromoto et al. (2004) mostraron que la FHA induce fracturamiento con morfología dúctil en el acero AISI 304L, y que la FHI induce, adicionalmente, fractura por clivaje. Su et al. (2000) observaron un modo de fractura mixto (dúctil y clivaje) en una aleación de $\mathrm{Ni}_{3} \mathrm{Al}$ bajo efecto da la $\mathrm{FHA}$, mientras que bajo la $\mathrm{FHI}$ el comportamiento fue dúctil o mixto, dependiendo de la concentración de hidrógeno.

El estudio de la FH en aceros de alta resistencia y baja aleación (HSLA) incluidos en la norma API $5 \mathrm{~L}$ es de particular interés para la industria petrolera, pues son ampliamente usados en ductos de transporte de hidrocarburos y tienen en su ambiente de trabajo las condiciones más favorables para el surgimiento de esos procesos de degradación: el uso de sistemas de protección catódica promueve la formación de hidrógeno y los hidrocarburos transportados generalmente contienen substancias que intensifican su adsorción y absorción en el material. Adicionalmente, el uso de aceros HSLA con resistencia cada vez mayor, aumentó la probabilidad de ocurrencia de la FH, pues está establecido que, cuanto mayor la resistencia mecánica de un acero, su susceptibilidad a la FH tiende a ser mayor (Interrante, 1982; Hardie et al., 2006; Beidokhti et al., 2009). Esta situación torna crítico el conocimiento de esa susceptibilidad para una correcta selección y uso seguro de esos materiales en estructuras que trabajan en ambientes que promueven la formación de hidrógeno. Dentro de esa línea, en este trabajo fue desarrollada una investigación experimental sobre el comportamiento mecánico de los aceros API $5 \mathrm{~L}$ grados X60 y X80, usados en la fabricación de tubos para oleoductos, con el propósito de estudiar su respuesta ante los procesos de FHA y FHI.

\section{PROCEDIMIENTO EXPERIMENTAL}

El estudio fue realizado para el acero $\mathrm{X} 60$, con micro-estructura compuesta de bandas de ferrita y perlita, típica de aceros laminados, y para el acero X80, con micro-estructura compuesta de ferrita y bainita, característica de aceros producidos por laminación controlada. Sus composiciones químicas están mostradas en la Tabla 1.

Tabla 1: Composición química de los aceros X60 y X80 (\%peso)

\begin{tabular}{|c|c|c|c|c|c|c|c|c|c|c|c|c|c|}
\hline & $\mathrm{C}$ & $\mathrm{Mn}$ & $\mathrm{Si}$ & $\mathrm{P}$ & $\mathrm{S}$ & $\mathrm{Al}$ & $\mathrm{Ti}$ & $\mathrm{Mo}$ & $\mathrm{Cu}$ & $\mathrm{V}$ & $\mathrm{Nb}$ & $\mathrm{Cr}$ & $\mathrm{Ni}$ \\
\hline $\mathrm{X} 60$ & 0.12 & 1.48 & 0.27 & 0.012 & 0.008 & 0.039 & 0.009 & 0.032 & 0.006 & 0.048 & 0.041 & -- & -- \\
\hline $\mathrm{X} 80$ & 0.084 & 1.61 & 0.23 & 0.01 & 0.011 & 0.035 & 0.015 & 0.17 & 0.029 & -- & -- & 0.135 & 0.011 \\
\hline
\end{tabular}

Para reproducir experimentalmente la FHA y la $\mathrm{FHI}$ fueron empleados dos procedimientos de hidrogenación y tres tipos de probetas fabricadas de la pared de tubos de ambos aceros maquinadas en la dirección de la laminación (Fig. 1): Tipo I (ASTM E8M), Tipo II (ASTM 
E8M+entalle) y Tipo III (ASTM E8M+entalle+extremidad alargada). Para cada condición se utilizaron tres probetas, que se ensayaron en tracción hasta su fractura a la temperatura ambiente.

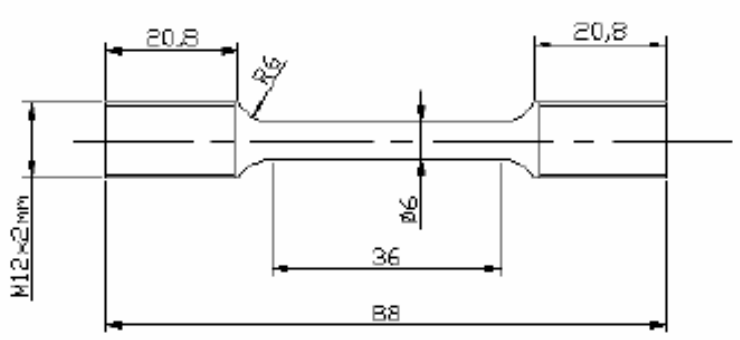

(a)

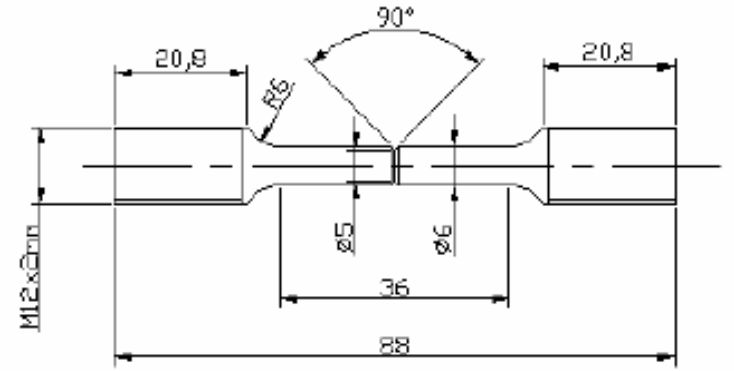

(b)

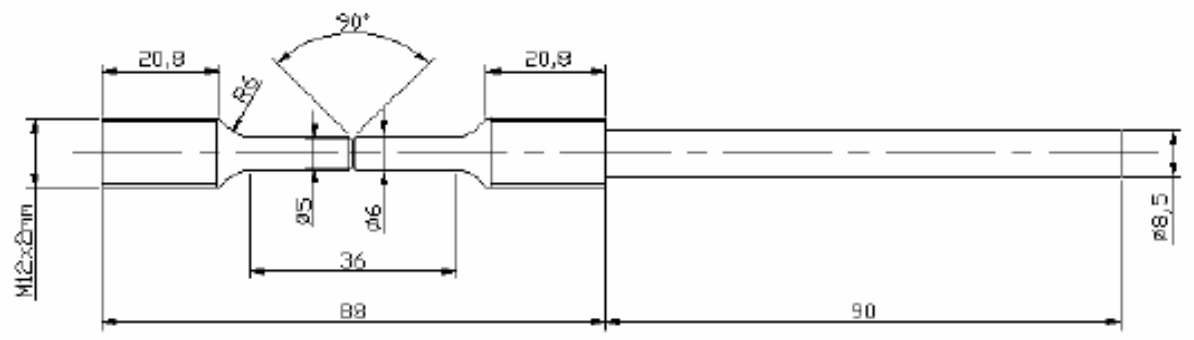

(c)

Fig. 1: Geometría y dimensiones (mm) de las probetas utilizadas, (a) Tipo I, (b) Tipo II, (c) Tipo III.

Inicialmente fue realizado un ensayo de polarización potenciodinámica para determinar el potencial y la densidad de corriente para la generación de hidrógeno. Con esa información, y conociendo su difusividad y solubilidad en los aceros estudiados, fueron estimados los tiempos de saturación. La difusividad fue determinada con el método de Devanathan y Stachurski (1962) en el equipo mostrado en la Fig. 2. Los ensayos fueron realizados en membranas metálicas de 3,14 $\mathrm{cm}^{2}$ de área $(A)$ y $1 \mathrm{~mm}$ de espesura $(L)$, de acuerdo a la norma ASTM G148-97.

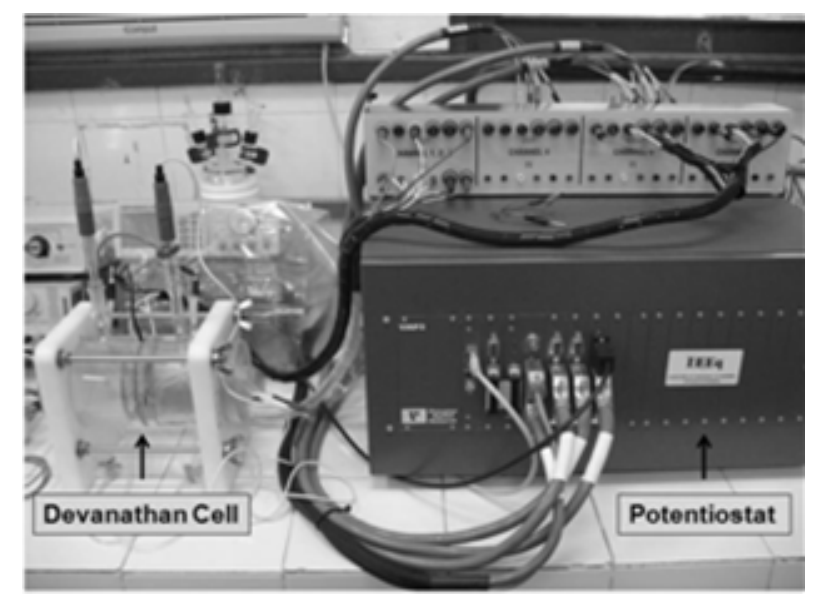

Fig. 2: Equipo utilizado en la determinación de la difusividad del hidrógeno en los dos aceros

El hidrógeno fue producido en la célula de carga, vía método galvanostático, con una densidad de corriente de $2,5 \mathrm{~mA} \cdot \mathrm{cm}^{-2}$ y una solución $0,1 \mathrm{~N}$ de $\mathrm{NaOH}$ con $2 \mathrm{mg}$ de $\mathrm{As}_{2} \mathrm{O}_{3}$, que evita la recombinación de hidrógeno. La célula de detección fue polarizada a un potencial constante de $+300 \mathrm{mV}_{\mathrm{SCE}}$ en una solución $0,1 \mathrm{~N}$ de $\mathrm{NaOH}$, para oxidar el hidrógeno permeado. La densidad de corriente asociada con esa oxidación fue monitoreada con el software EC-LAB v.10.02, controlado por un potenciostato multicanal PAR modelo VMP3 hasta que fue alcanzado el estado estacionario. Las soluciones de las células fueron desoxigenadas con nitrógeno puro antes y durante el ensayo. La difusividad $(D)$, permeabilidad $(P)$ y solubilidad $(S)$ fueron calculadas con la curva de permeación de hidrógeno y las ecuaciones de Boes y Züchner (1976): 
$P=J_{\infty} L$

$t_{b}=0,76 \frac{L^{2}}{\pi^{2} D}$

$t_{L}=\frac{L^{2}}{2 D}$

$S=\frac{P}{D}$

$t_{b}$ es el tiempo necesario para que los primeros átomos de hidrógeno arriben al lado de detección. $t_{L}$ es el punto de intersección del eje de las abscisas con la recta tangente a la curva $\int i_{L} d t$, cuya pendiente permanece constante cuando es establecido un flujo en estado estacionario, $J_{\infty}$.

Para reproducir la condición de la FHA, el hidrógeno fue introducido en las probetas Tipo I y Tipo II vía difusión generada por el gradiente de concentración entre la superficie y la matriz metálica. El hidrógeno fue producido potenciostáticamente en la superficie de la probeta con una fuente de poder ICEI-PS7000 y una solución $0,1 \mathrm{M}$ de $\mathrm{NaOH}$ con $2 \mathrm{mg}$ de $\mathrm{As}_{2} \mathrm{O}_{3}$ a la temperatura ambiente; como ánodo fue utilizado un electrodo FISHER de Pt-Ir. La carga de hidrógeno fue realizada por $24 \mathrm{~h}$ con una densidad de corriente de $2,5 \mathrm{~mA} \cdot \mathrm{cm}^{-2}$, sin tensión mecánica. Posteriormente, las probetas fueron sumergidas en una solución de $\mathrm{Cu}_{2} \mathrm{SO}_{4}$ para reducir la desorción del hidrógeno (Zampronio, 1995), posibilitando así que su concentración permanezca prácticamente invariable hasta el inicio de los ensayos de tracción, realizados inmediatamente después de concluida la carga de hidrógeno.

Para reproducir la condición de la $\mathrm{FHI}$, el hidrógeno fue introducido catódicamente en las probetas Tipo III a la temperatura ambiente, con el mismo potencial y la misma solución empleados en el procedimiento anterior. La metodología de carga de hidrógeno utilizada fue la propuesta por Tiwari et al. (2000): (a) empleo de una probeta entallada con uno de sus extremos alargado (Tipo III), que forma el cátodo de la célula electroquímica; (b) hidrogenación durante $24 \mathrm{~h}$ y $48 \mathrm{~h}$ bajo tracción con una tensión del $65 \%$ del límite de fluencia del material, a fin de permitir una concentración de hidrógeno por sobre su límite de solubilidad; (c) estabilización de esa concentración durante 24h adicionales al tiempo de hidrogenación, para posibilitar que, al final de ese período, la mayor parte del hidrógeno introducido en el acero difunda para fuera del reticulado y que el restante permanezca aprisionado en sus trampas irreversibles. En este procedimiento, en el que fue utilizado un dispositivo desarrollado en la UFCG (Fig. 3), el hidrógeno fue generado en la extremidad alargada de la probeta y la hidrogenación promovida por los gradientes de concentración y de tensiones, generados en la extremidad alargada y en el entalle, respectivamente.
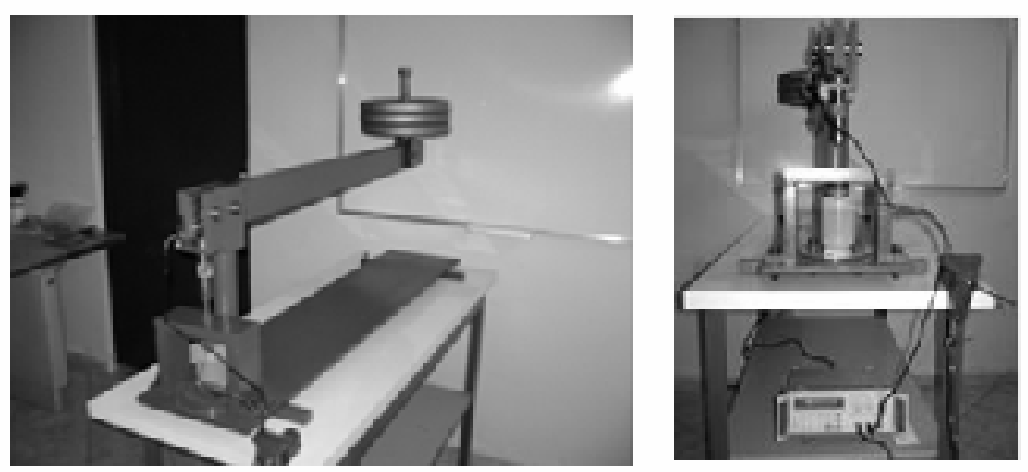

Fig. 3: Dispositivo utilizado en el proceso de hidrogenación para reproducir la FHI. 
Los ensayos de tracción fueron realizados a la temperatura ambiente en una máquina universal de ensayos INSTRON modelo 5582, a una tasa de deformación de $2,5 \times 10^{-5} \cdot \mathrm{s}^{-1}$, de acuerdo con la norma ASTM E8M. Para evaluar la influencia del hidrógeno en el modo de fractura, la superficie fracturada de las probetas fue estudiada con un microscopio electrónico de barrido (SEM).

\section{RESULTADOS Y DISCUSIÓN}

\section{Polarización potenciodinámica}

Las curvas de polarización potenciodinámica para los dos aceros están mostradas en las Figs. 4(a) y 4(b), donde puede observarse que el potencial para producir una densidad de corriente de $2,5 \mathrm{~mA} / \mathrm{cm}^{2}$ fue de aproximadamente $-1,52 \mathrm{~V}_{\text {SCE }}$ para el acero $\mathrm{X} 60$ y de $-1,4 \mathrm{~V}_{\text {SCE }}$ para el acero $\mathrm{X} 80$.

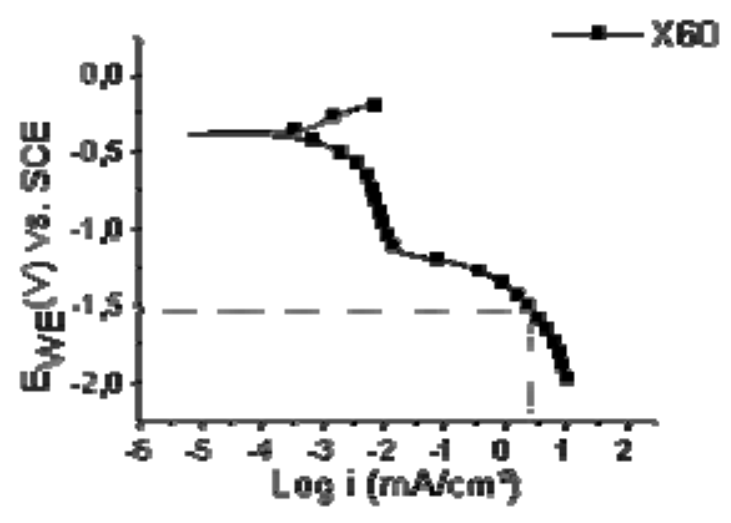

(a)

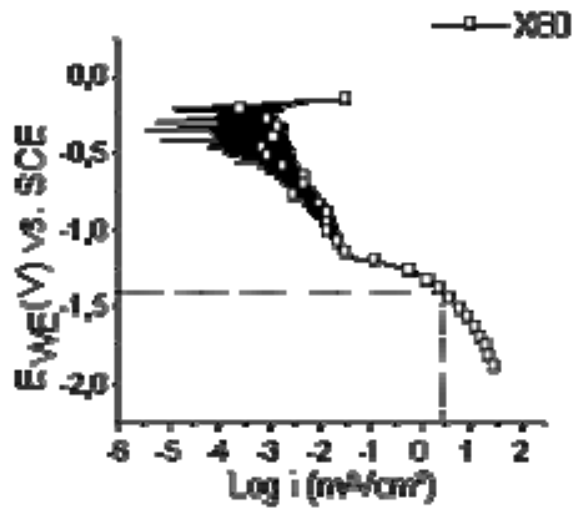

(b)

Fig. 4: Curvas de polarización potenciodinámica de los aceros (a) X60; (b) X80.

\section{Permeación electroquímica}

Las curvas de permeación de hidrógeno en los aceros estudiados están mostradas en la Fig. 5.

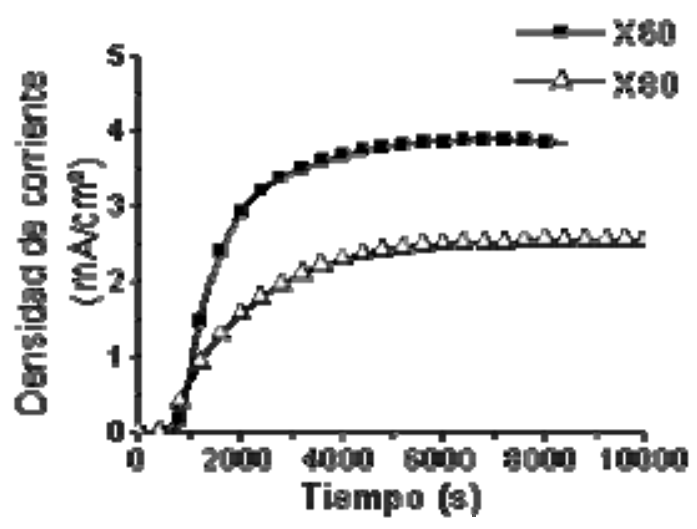

Fig. 5: Curvas de permeación de hidrógeno de los aceros X60 y X80.

Los resultados de la difusividad aparente, solubilidad aparente y permeabilidad del hidrógeno en los dos aceros, para las condiciones de ensayo, están mostrados en la Tabla 2.

El acero X80 presentó una difusividad mayor, una permeabilidad ligeramente inferior y una solubilidad mucho menor que las del acero X60, diferencias que pueden ser atribuidas a la microestructura y/o a la composición química de esos aceros. La diferencia entre las difusividades puede ser explicada por el contenido de carbono, que está asociado al número de carburos en el acero. Como éstos dificultan la difusión de hidrógeno, fue establecido que aceros con alto 
contenido de carbono presentan menores difusividades que aquellos con contenido menor, y que el tipo de distribución de carburos también puede afectar la difusividad. Como en el acero X80 esa distribución es menos uniforme, el hidrógeno tiene una mayor difusividad que en el acero X60, aunque el tiempo para alcanzar el estado estacionario sea mayor. Esto permite explicar la mayor capacidad de atrapamiento de hidrógeno mostrada por el acero X60 (Luu y Wu, 1996). Con respecto a la diferencia de permeabilidades, ya fue establecido que la micro-estructura bainítica presenta una menor permeabilidad al hidrógeno que la perlítica (Parvathavarthini et al., 1999).

Tabla 2: Valores medios de los parámetros obtenidos de los ensayos de permeación de hidrógeno.

\begin{tabular}{|l|c|c|c|}
\hline \multicolumn{1}{|c|}{ Acero } & $\begin{array}{c}\text { Difusividad } \\
\left(10^{-10} \mathrm{~m}^{2} \cdot \mathrm{s}^{-1}\right)\end{array}$ & $\begin{array}{c}\text { Permeabilidad } \\
\left(10^{-10} \mathrm{molH} \cdot \mathrm{m}^{-1} \cdot \mathrm{s}^{-1}\right)\end{array}$ & $\begin{array}{c}\text { Solubilidad } \\
\left(\mathrm{molH} \cdot \mathrm{m}^{-3}\right)\end{array}$ \\
\hline $\mathrm{X} 60$ & $2.11 \pm 0.38$ & $3.53 \pm 0.82$ & $1.66 \pm 0.08$ \\
\hline $\mathrm{X} 80$ & $3.37 \pm 0.15$ & $3.01 \pm 0.16$ & $0.89 \pm 0.06$ \\
\hline
\end{tabular}

\section{Ensayos de tracción en condiciones de FHA}

La Fig. 6 muestra las curvas tensión-deformación de los aceros X60 y X80, probetas Tipo I, sin y con hidrógeno. El resumen de las propiedades mecánicas determinadas en los ensayos se muestra en la Tabla 3. Puede verificarse que el acero X60 hidrogenado presentó un aumento de aproximadamente $2,04 \%$ en la ductilidad y disminuciones en los límites de fluencia $\left(\sigma_{y}\right)$ y de resistencia a la tracción ( $\left.\sigma_{U T S}\right)$, evidenciando que sufrió un reblandecimiento. El acero X80 registró un efecto contrario, presentando una disminución del $1,80 \%$ en su ductilidad y pequeños incrementos en los límites de fluencia y de resistencia a la tracción.

La susceptibilidad de los aceros a la FH puede ser evaluada a través de la determinación de sus propiedades mecánicas en tracción y su comparación con las propiedades determinadas en pruebas en condiciones de FH. Esa comparación proporciona Índices de Fragilización por Hidrógeno (IFH) que, cuando próximos de la unidad, indican baja susceptibilidad (o resistencia) y, cuanto más próximos del cero, susceptibilidad más alta. Esa susceptibilidad también puede ser relacionada con el cambio en los modos de fractura (Tsay et al., 2000). Los IFH obtenidos luego de la comparación de la ductilidad de los aceros ensayados, con y sin hidrógeno, fueron: 1,02 para el X60 y 0,98 para el X80, lo que permite afirmar que, para las condiciones del ensayo realizado, el acero X60 es resistente a la FHA y que el X80 es poco susceptible a ese fenómeno.

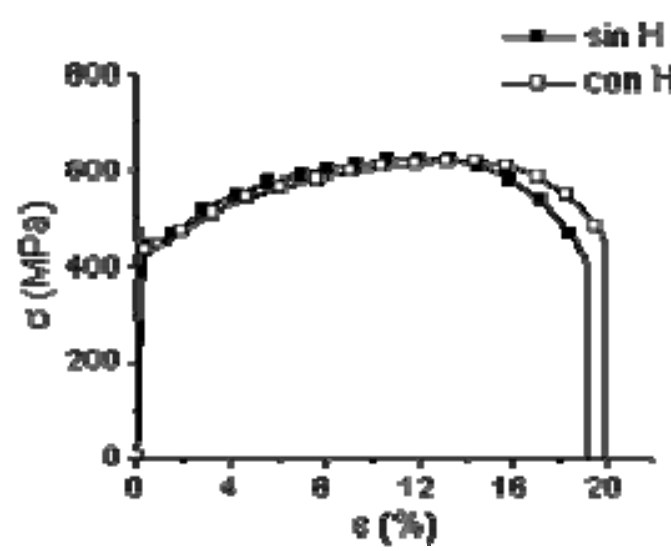

(a)

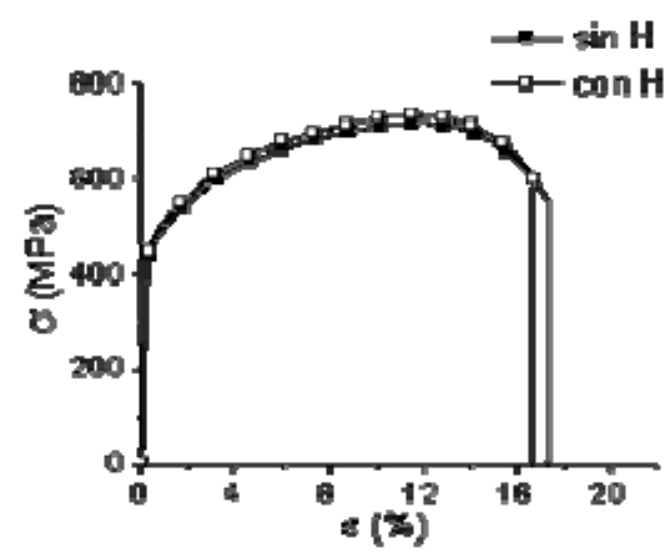

(b)

Fig. 6: Curvas tensión-deformación probetas Tipo I sin y con hidrógeno. Acero (a) X60, (b) X80.

La Fig. 7 muestra las curvas tensión-deformación de los aceros X60 y X80, probetas Tipo II, sin y con hidrógeno. El resumen de las propiedades mecánicas determinadas en los ensayos está mostrado en la Tabla 4. Los resultados obtenidos permiten verificar que, a diferencia de los ensayos con las probetas del Tipo I, ambos aceros presentaron una disminución en la ductilidad, lo que muestra que el efecto del hidrógeno es intensificado con la presencia del entalle en la 
probeta. Esto puede ser explicado por el hecho de que la acumulación del hidrógeno en regiones con elevados niveles de concentración de tensiones es un factor de intensificación del proceso de FH (Oriani, 1978). Adicionalmente, el hidrógeno puede disminuir la tensión de fractura en probetas entalladas, siendo esta disminución proporcional a la cantidad de hidrógeno presente. Es así que una reducción en esa tensión es también un indicador de la susceptibilidad a la FH del acero ensayado (Wang et al., 2007).

Tabla 3: Propiedades mecánicas de los aceros X60 y X80. Probetas Tipo I sin y con hidrógeno.

\begin{tabular}{|l|c|c|c|c|}
\hline \multirow{2}{*}{ Parámetro } & \multicolumn{2}{|c|}{ X60 } & \multicolumn{2}{c|}{ X80 } \\
\cline { 2 - 5 } & Sin hidrógeno & Con hidrógeno & Sin hidrógeno & Con hidrógeno \\
\hline$\sigma_{\mathrm{Y}}(\mathrm{MPa})$ & $462 \pm 10.8$ & $450 \pm 8.5$ & $488 \pm 4.7$ & $491 \pm 5.4$ \\
\hline$\sigma_{\text {UTS }}(\mathrm{MPa})$ & $629 \pm 4.2$ & $627 \pm 4.7$ & $720 \pm 3.2$ & $722 \pm 9.5$ \\
\hline$\varepsilon(\%)$ & $19.08 \pm 0.14$ & $19.47 \pm 0.12$ & $16.69 \pm 0.24$ & $16.39 \pm 0.09$ \\
\hline
\end{tabular}

Fue obtenido un IFH del 0,87 para los dos aceros, lo que permite concluir que, para las condiciones del ensayo realizado, ambos presentan una moderada susceptibilidad a la FHE.

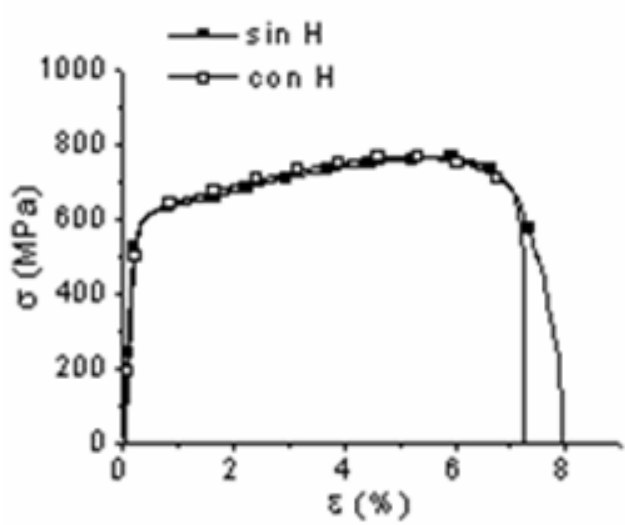

(a)

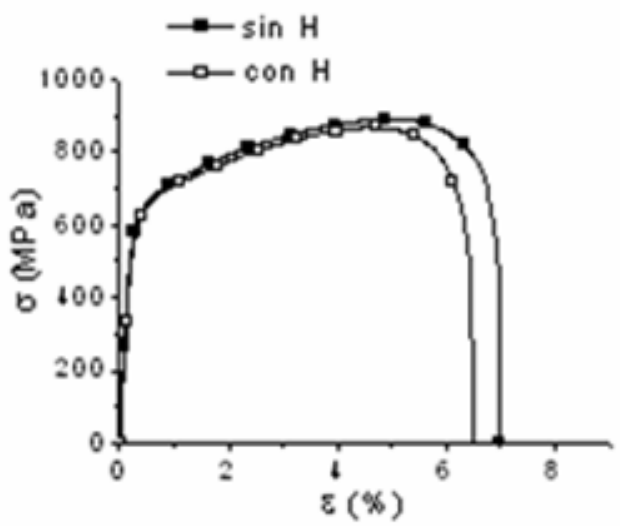

(b)

Fig. 7: Curvas tensión-deformación probetas Tipo II sin y con hidrógeno. Acero (a) X60, (b) X80.

Tabla 4: Propiedades mecánicas, aceros X60 y X80. Probetas Tipo II sin y con hidrógeno.

\begin{tabular}{|l|c|c|c|c|}
\hline \multirow{2}{*}{ Parámetro } & \multicolumn{2}{|c|}{ X60 } & \multicolumn{2}{c|}{ X80 } \\
\cline { 2 - 5 } & Sin hidrógeno & Con hidrógeno & Sin hidrógeno & Con hidrógeno \\
\hline$\sigma_{U T S}(\mathrm{MPa})$ & $772 \pm 6.7$ & $766 \pm 4.8$ & $881 \pm 20.8$ & $868 \pm 11.1$ \\
\hline$\varepsilon(\%)$ & $7.89 \pm 0.38$ & $6.85 \pm 0.35$ & $7.07 \pm 0.35$ & $6.18 \pm 0.49$ \\
\hline
\end{tabular}

La susceptibilidad de los aceros HSLA a los efectos del hidrógeno ha sido estudiada por varios investigadores, como Hardie et al. (2006), que trabajaron con aceros X60, X80 y X100; Trasatti et al. (2005) y Cwiek (2005), con aceros X80; Beidokhti et al. (2009) y Torres-Islas et al. (2005) con aceros X70; Vianna (2005) y Araújo et al. (2009) con aceros X60; Domizzi et al. (2001) con ocho tipos de aceros, incluidos dos X60. La mayoría de los trabajos citados reporta pérdida de ductilidad de los aceros en diversas condiciones de ensayo, diferentes de las aquí utilizadas; Vianna (2005) y Araújo et al. (2009) reportan un ligero aumento de la ductilidad del acero en condiciones de ensayo semejantes a las empleadas aquí. Domizzi et al. (2001) no presentan ninguna observación del efecto del hidrógeno sobre la ductilidad de los aceros estudiados.

Esta diferencia con los resultados reportados en la literatura científica puede atribuirse a que, aparte de las diferencias en las condiciones de los ensayos realizados, la composición química de los aceros X60 y X80 estudiados en esos trabajos no es exactamente la misma. A pesar de que la composición química de esos aceros se encuentra definida por la norma API 5L, en ella solo figuran las cantidades máximas permitidas, lo que da lugar a variaciones de composición dependiendo del fabricante. Como es sabido, la presencia de ciertos elementos en la micro- 
estructura de un acero condiciona el comportamiento del hidrógeno en el reticulado; la diferencia en sus cantidades podrá resultar, por lo tanto, en la variación del comportamiento del hidrógeno en aceros fabricados bajo la misma norma, con reflejos directos sobre su respuesta mecánica.

\section{Ensayos de tracción en condiciones de FHI}

La Fig. 8 muestra las curvas tensión-deformación de los aceros X60 y X80, probetas Tipo III sin hidrógeno e hidrogenadas bajo tensión durante $24 \mathrm{~h}$ y $48 \mathrm{~h}$. El resumen de las propiedades mecánicas está en la Tabla 5. Los IFH para el acero X60 fueron 0,98 con carga de hidrógeno por $24 \mathrm{~h}$ y 1,08 con carga de hidrógeno por $48 \mathrm{~h}$. Para el acero X80, los IFH fueron 1,05 con carga por $24 \mathrm{~h}$ y 1,02 con carga por $48 \mathrm{~h}$. Esto lleva a afirmar que, para las condiciones empleadas en los ensayos, los dos aceros son resistentes a la $\mathrm{FHI}$, comportamiento que puede ser explicado por sus composiciones químicas y micro-estructuras características: los dos aceros contienen elementos micro-aleantes de efecto benéfico en el control de la $\mathrm{FH}$, como elementos formadores de carburos, usados en el refinado de los granos, endurecimiento por precipitación y en la mejora de la tenacidad, que constituyen trampas efectivas de hidrógeno.

Es bien sabido que las aleaciones desarrolladas para resistir al daño causado por el hidrógeno deben tener un número apreciable de trampas irreversibles de hidrógeno bien distribuidas por toda su micro-estructura (OTO, 1999). Adicionalmente, aceros con contenido de $\mathrm{Nb}$ presentan buena resistencia a la $\mathrm{FH}$ debido a que forman partículas de NbC (Gojic et al., 1998); precipitados de $\mathrm{Mo}_{2} \mathrm{C}, \mathrm{VC}$ y TiC también aumentan esa resistencia. Por lo tanto, como la cantidad de hidrógeno que puede ser liberada por la micro-estructura es la que se torna más importante durante los procesos de $\mathrm{FH}$, el hidrógeno atrapado reversiblemente es más perjudicial que el atrapado de forma irreversible (Luppo y Garcia, 1991). Este efecto puede ser observado en los aceros ensayados aquí, y probablemente se deba al método de hidrogenación empleado, que, asociado a la tensión aplicada, intenta reproducir el fenómeno de $\mathrm{FHI}$, posibilitando que una gran cantidad de hidrógeno permanezca aprisionada en las trampas irreversibles del acero.

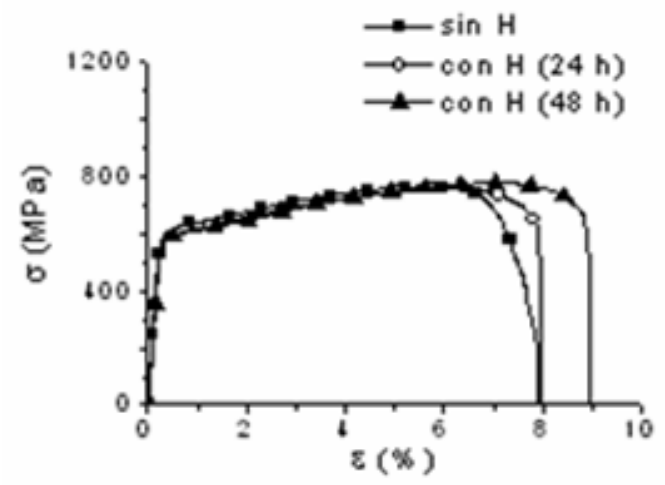

(a)

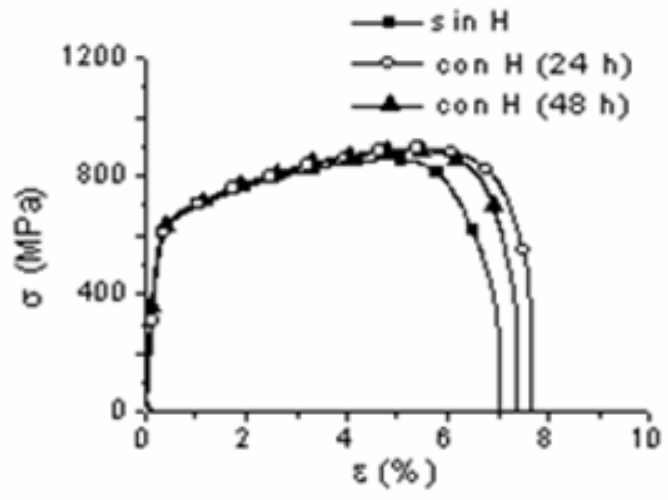

(b)

Fig. 8: Curvas tensión-deformación para probetas Tipo III sin y con hidrógeno cargado bajo tensión por $24 \mathrm{~h}$ y $48 \mathrm{~h}$. Acero (a) X60, (b) X80.

A pesar de que existen publicaciones científicas relacionadas con la FH en aceros HSLA, ellas se refieren a la FHA, no habiendo sido posible encontrar artículos con estudios específicos sobre la $\mathrm{FHI}$ en estos aceros. Estudios sobre los efectos de la FHI en el acero Maraging T250 y un acero dulce, realizados bajo las mismas condiciones de hidrogenación empleadas aquí, registran disminuciones en sus ductilidades, seguidas de un endurecimiento (Tiwari et al., 2000).

\section{Caracterización fractográfica SEM - fracturas por FHA}

Las figuras 9 (a) y 9 (b) muestran imágenes de la superficie de fractura de probetas Tipo I de acero X60 sin y con hidrógeno. En ellas se observa que el modo de fractura predominante en las dos condiciones es dúctil por ruptura de dimples, comportamiento característico de ese tipo de aceros. 
En la figura 9(b), que corresponde al material hidrogenado, puede observarse que los dimples son mucho más pequeños y que aparecen en mayor cantidad que los encontrados en el material libre de hidrógeno. Esos pequeños dimples son formados por la activación de un gran número de sitios de nucleación en los que los microvacíos coalescen antes de tener la oportunidad de crecer a tamaños mayores. Este efecto es causado por la gran cantidad de átomos de hidrógeno absorbidos por el material, que ocasiona que los dimples fallen "precozmente" y que aumenten su cantidad.

Tabla 5. Propiedades mecánicas del acero X60. Probetas Tipo III sin y con hidrógeno (24h y 48h).

\begin{tabular}{|l|c|c|c|}
\hline \multirow{2}{*}{ Parámetro } & \multicolumn{3}{|c|}{ X60 } \\
\cline { 2 - 4 } & Sin hidrógeno & $\begin{array}{c}\text { Con hidrógeno } \\
\text { (bajo tensión 24 } \mathrm{h} \text { ) }\end{array}$ & $\begin{array}{c}\text { Con hidrógeno } \\
\text { (bajo tensión 48 } \mathrm{h} \text { ) }\end{array}$ \\
\hline$\sigma_{\mathrm{UTS}}(\mathrm{MPa})$ & $772 \pm 6.7$ & $773 \pm 18.7$ & $785 \pm 5.4$ \\
\hline$\varepsilon(\%)$ & $7.89 \pm 0.38$ & $7.70 \pm 0.20$ & $8.51 \pm 0.31$ \\
\hline \hline \multirow{3}{*}{$\sigma_{\mathrm{UTS}}(\mathrm{MPa})$} & \multicolumn{3}{|c|}{$\mathrm{X80}$} \\
\hline$\varepsilon(\%)$ & $881 \pm 20.8$ & $901 \pm 10.0$ & $891 \pm 0.8$ \\
\cline { 2 - 4 } & $7.07 \pm 0.35$ & $7.40 \pm 0.18$ & $7.19 \pm 0.05$ \\
\hline
\end{tabular}

Las figuras 9 (c) y 9 (d) muestran imágenes de la superficie de fractura de probetas Tipo I de acero X80 sin y con hidrógeno, en las que se verifica que la morfología de fractura predominante en ambas condiciones también es dúctil por ruptura de dimples. A pesar de que el proceso de carga de hidrógeno permitió llegar a una condición de saturación, la presencia de pocas fisuras en la superficie de las probetas hidrogenadas explica la pequeña pérdida de ductilidad mostrada por este acero y, consecuentemente, su baja susceptibilidad a la $\mathrm{FH}$, dado que la formación de esas fisuras es atribuida a la acción del hidrógeno.

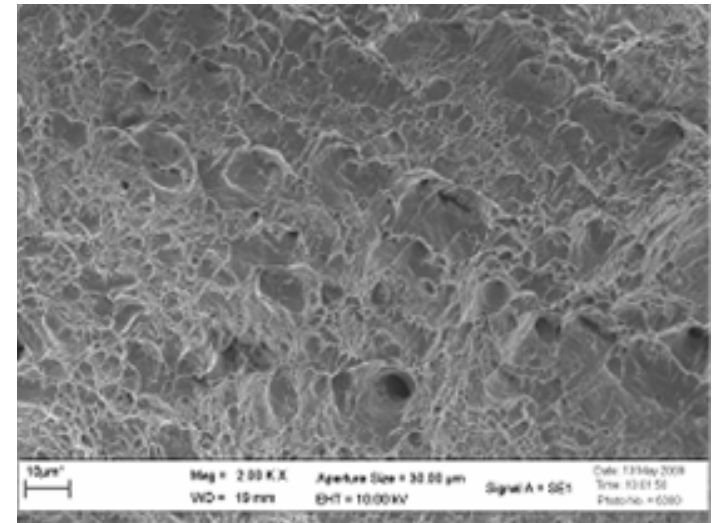

(a)

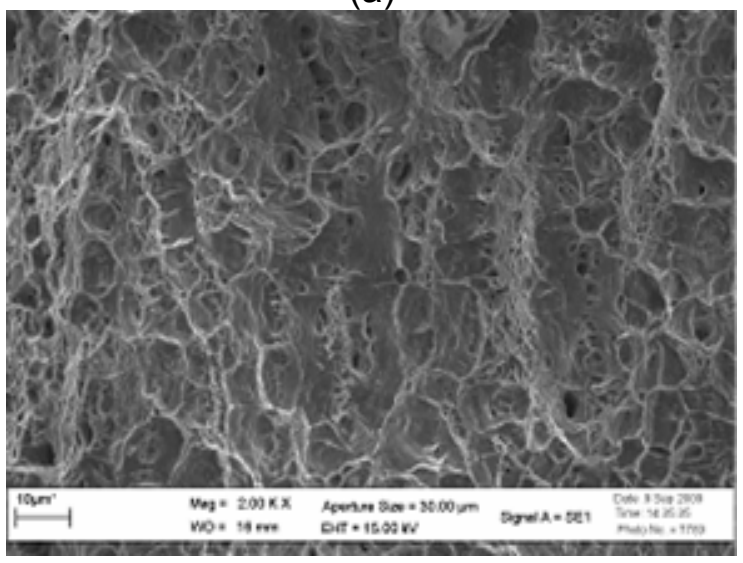

(c)

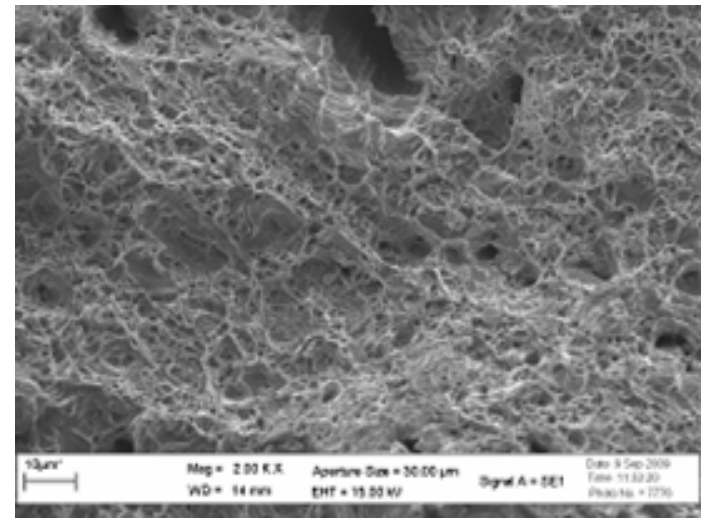

(b)

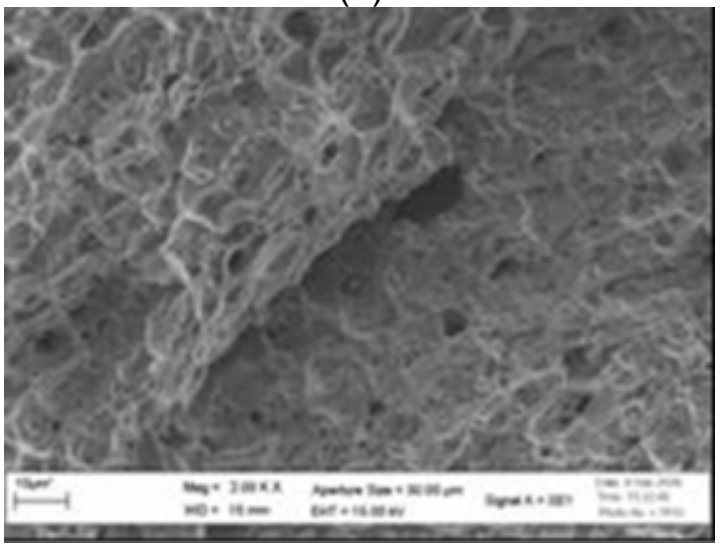

(d)

Fig. 9. Imagen SEM de la superficie de fractura de probetas Tipo I, aceros (a) X60 sin hidrógeno; (b) X60 con hidrógeno; X80 sin hidrógeno; (b) X80 con hidrógeno. 
Las imágenes de la superficie de fractura de la región próxima al centro de las probetas Tipo II de los aceros X60 y X80 con hidrógeno están mostradas en las Figs. 10(a) y 10(b), donde se observa que el modo de fractura en ambos casos es predominantemente dúctil por ruptura de dimples. A diferencia de los ensayos realizados con las probetas Tipo I, la superficie de fractura del material hidrogenado presenta fisuras, a pesar de que el método de hidrogenación fue el mismo. La diferencia en los IFH obtenidos, se explica por la elevada concentración de hidrogeno en la región al frente del entalle, que puede reducir aún más la fuerza cohesiva del material, y tornar al acero más susceptible a la FHA en esta condición.

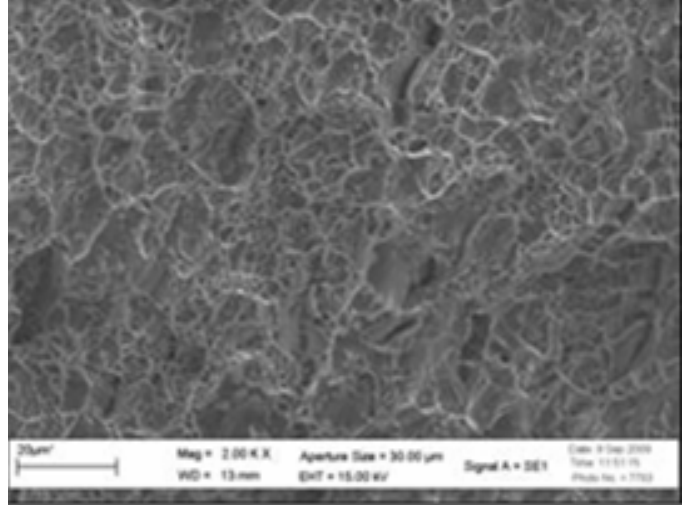

(a)

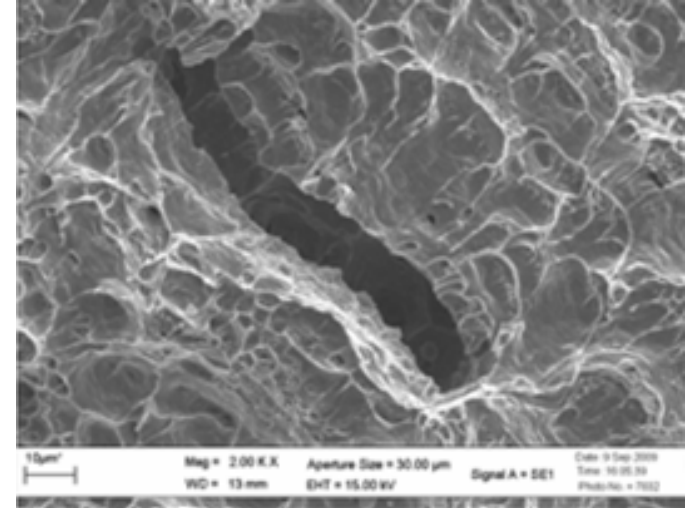

(b)

Fig. 10. Imagen SEM de la superficie de fractura de probetas Tipo II hidrogenadas: (a) X60; (b) X80.

Una particularidad de efecto del hidrógeno sobre el acero X80 en la condición de este ensayo, es la existencia de regiones con modo de fractura por cuasi clivaje, característico de procesos de $\mathrm{FH}$. Estas regiones, localizadas en las proximidades de las fisuras, afectan también la ductilidad local y explican la mayor susceptibilidad de este acero a la FHE cuando existe un concentrador de tensiones.

\section{Caracterización fractográfica SEM - fracturas por FHI}

Las Figs. 11(a) y 11(b) muestran imágenes de las superficies de fractura de la región próxima al centro de las probetas Tipo III de los aceros X60 y X80 con hidrógeno cargado por 48h bajo tensión, donde se observa que el modo de fractura predominante en las dos condiciones es dúctil por ruptura de dimples. Los resultados obtenidos para los ensayos de ambos aceros en condición de $\mathrm{FHI}$, asociados a la inexistencia de alteraciones apreciables en los modos de fractura de las probetas sin y con hidrógeno, llevan a concluir que son resistentes a ese tipo de fragilización, lo que se explica, como mostrado antes, por la habilidad que tienen de atrapar el hidrógeno con altas energías de aprisionamiento.

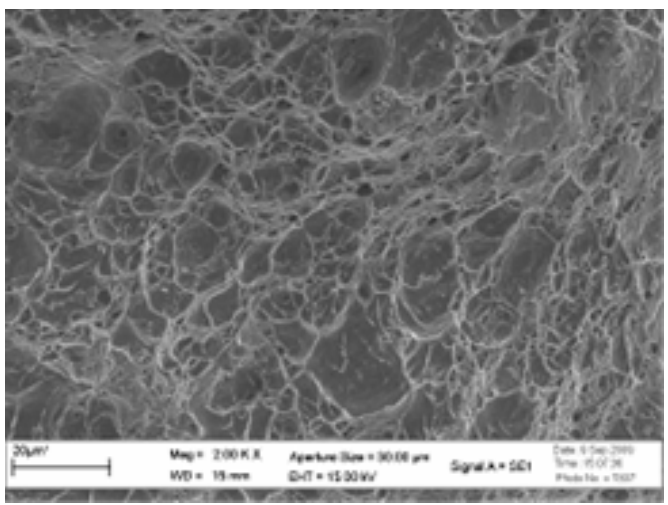

(a)

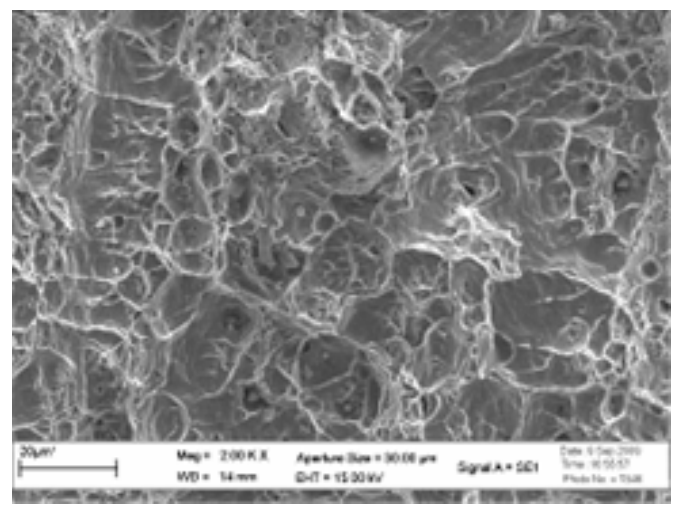

(b)

Fig. 11. Imagen SEM de la superficie de fractura de probetas Tipo III: (a) X60 con hidrógeno (48h); (b) X80 con hidrógeno (48h). 


\section{CONCLUSIONES}

La morfología de la superficie de fractura de las probetas Tipo I y Tipo II de los aceros X60 y X80, sin hidrógeno y bajo efecto de la FHA, permaneció predominantemente dúctil por ruptura de dimples, existiendo entretanto, algunas regiones de fractura por cuasi clivaje en las probetas entalladas del acero X80. La variación casi inexistente en los modos de fractura y los IFH obtenidos para cada condición, permiten concluir que, para las condiciones empleadas en los ensayos, ambos aceros son poco susceptibles a la FHA, y que esa susceptibilidad se torna moderada con la presencia de un concentrador de tensiones, que intensifica la acción del hidrógeno.

La ausencia de variaciones en la morfología de fractura de las probetas Tipo III, y los IFH obtenidos en las condiciones empleadas en los ensayos, permiten concluir que los aceros X60 y $\mathrm{X} 80$ no presentan susceptibilidad a la $\mathrm{FHI}$.

Los aceros $\mathrm{X} 60$ y $\mathrm{X} 80$ son poco afectados por la acción del hidrógeno, sea de origen interno o ambiental. Esta característica puede ser atribuida a la presencia de elementos componentes de su micro-estructura que atrapan el hidrógeno con altas energías de aprisionamiento, y que reducen su capacidad de difundir y de generar procesos de degradación en otras regiones del reticulado, como micro-vacíos y puntas de micro-fisuras, altamente sensibles a su efecto deletéreo.

\section{AGRADECIMIENTOS}

Los autores agradecen a la FINEP/CTPETRO/CNPq/PETROBRAS/RPCMod y al programa ANP/UFCG-PRH-25 por financiar este trabajo de investigación, así como a los laboratorios LEEQ/UFCG, LaMMEA/UFCG y NEPEM/UFPB por su contribución durante la fase experimental.

\section{REFERENCIAS}

Araújo, B.A., G.D. Travassos, E.O. Vilar, J.P. Carrasco y A.A. Silva, A Study of Internal Hydrogen Embrittlement and Environmental Hydrogen Embrittlement of API $5 \mathrm{~L} \mathrm{X60} \mathrm{Steel,} \mathrm{Memorias} \mathrm{del} 20^{\circ}$ Congreso Brasileño de Ingenieria Mecánica, Gramado, Brasil, 15 al 20 de noviembre (2009).

ASM Metals Handbook, Corrosion: Fundamentals, Testing, and Protection, v 13A, $9^{a}$ edición, ASM International, Materials Park, OH, EUA (1987).

Beidokhti, B., A. Dolati y A.H. Koukabi, Effects of Alloying Elements and Microstructure on the Susceptibility of the Welded HSLA Steel to Hydrogen-Induced Cracking and Sulfide Stress Cracking, Materials Science and Engineering A: 507, 167-173 (2009).

Boes, N. y H. Züchner, Electrochemical Methods for Studying Diffusion, Permeation and Solubility of Hydrogen in Metals, Journal of the Less-Common Metals: 49, 223-246 (1976).

Cwiek, J., Hydrogen Assisted Cracking of High-Strength Weldable Steels in Sea-Water, Journal of Materials Processing Technology: 164(165), 1007-1013 (2005).

Devanathan, M.A.V. y Z. Stachurski. The Adsorption and Diffusion of Electrolytic Hydrogen in Palladium, Proceedings of the Royal Society: A270, 90-102 (1962).

Domizzi, G., G. Anteri y J. Ovejero-García, Influence of Sulphur Content and Inclusion Distribution on the Hydrogen Induced Blister Cracking in Pressure Vessel and Pipeline Steels, Corrosion Science: 43, 325-339 (2001).

Eliaz, N., A. Shachar, B. Tal y D. Eliezer, Characteristics of HE, SCC in High Strength Steels, Engineering Failure Analysis: 9, 167-184 (2002). 
Gojic, M., L. Kosec y L. Vehovar, The susceptibility to Sulphide Stress Cracking of Low Alloy Steels, Materials and Corrosion: 49, 27-33 (1998).

Hardie, D., E.A. Charles y A.H. Lopez, Hydrogen Embrittlement of High Strength Pipeline Steels, Corrosion Science: 48, 4378-4385 (2006).

Interrante, C.G., Basic Aspects of the Problems of Hydrogen in Steels, ASM, 3-16 (1982).

Kuromoto, N.K., A.S. Guimarães y C.M. Lepienski, Superficial and Internal Hydrogenation Effects on the Fatigue Life of Austenitic Steels, Materials Science and Engineering A: 381, 216-222 (2004).

Luppo, M. y O.J. Garcia, The influence of microstructure on the trapping and diffusion of hydrogen in a low carbon steel, Corrosion science: 32 (10), 1125-1136 (1991).

Luu, W.C. y J.K. Wu, The influence of microstructure on hydrogen transport in carbon steels. Corrosion Science: 38 (2), 239-245 (1996).

OTO - Offshore Tecnology Report, A Review of the Effects on the Hydrogen Embrittlement of High Strength Offshore Steels, Healt and Safety Executive (1999).

Oriani, R.A., Hydrogen embrittlement of steels, Ann. Rev. Mater. Sci.: 8, 327-57 (1978).

Parvathavarthini, N., S. Saroja y R.K. Dayal, Infuence of Microstructure on the Hydrogen Permeability of 9\%Cr-1\%Mo Ferritic Steel, Journal of Nuclear Materials: 264, 35-47 (1999).

Su, J.Q., S.J. Gao y Z.Q. Hu, Environmental and Internal Hydrogen Embrittlement of a Directionally Solidified Ni-rich $\mathrm{Ni}_{3} \mathrm{Al}$ Intermetallics, Journal of Materials Science: $35,1433-1438$ (2000).

Tiwari, G.P. y otros seis autores, A Study of Internal Hydrogen Embrittlement of Steels, Materials Science and Engineering A: 286, 269-281 (2000).

Torres-Islas, A., V.M. Salinas-Bravo, J.L. Albarran y J.G. Gonzalez-Rodriguez, Effect of Hydrogen on the Mechanical Properties of X-70 Pipeline Steel in Diluted NaHCO3 Solutions at Different Heat Treatments, International Journal of Hydrogen Energy: 30, 1317-1322 (2005).

Trasatti, S.P., E. Sivieri y F. Mazza, Susceptibility of a X80 Steel to Hydrogen Embrittlement, Materials and Corrosion: 56(2), 111-117 (2005).

Tsay, L.W., Z.W. Lin, R.K. Shiue, y C. Chen, Hydrogen Embrittlement Susceptibility of LaserHardened 4140 Steel, Materials Science and Engineering A: 290, 46-54 (2000).

Vianna C.S., Comportamento Mecânico do Aço API $5 L$ X-60 com e sem Hidrogênio, Tesis de Maestría, Depto. de Ing. Metalúrgica y de Materiales, UFRJ, Rio de Janeiro, Brasil (2005).

Wang, M., E. Akiyama y K. Tsuzaki, Effect of hydrogen on the fracture behavior of high strength steel during slow strain rate test, Corrosion Science: 49, 4081-4097 (2007).

Woodtli, J. y R. Kieselbach, Damage due to Hydrogen Embrittlement and Stress Corrosion Cracking, Engineering Failure Analysis: 7, 427-450 (2000).

Zampronio, M.A., Revestimento em Aço para Limitar a Contaminação por Hidrogênio, Tesis de Doctorado, Depto. de Ing. Metalúrgica y de Materiales, UFRJ, Rio de Janeiro, Brasil (1995). 